Exp Brain Res (1994) 97:477-486

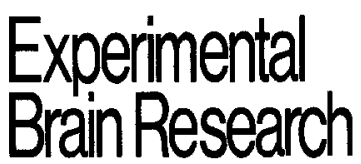

(C) Springer-Verlag 1994

\title{
Temporal stability of the action-perception cycle for postural control in a moving visual environment
}

\author{
T.M.H. Dijkstra ${ }^{1}$, G. Schöner ${ }^{2}$, C.C.A.M. Gielen ${ }^{1}$ \\ ${ }^{1}$ Laboratory of Medical Physics and Biophysics, University of Nijmegen, P.O. Box 9101, 6500 HB, Nijmegen, The Netherlands \\ ${ }^{2}$ Institut für Neuroinformatik, Ruhr-Universität Bochum, Postfach 102148, ND04, D-44801, Bochum, Germany
}

28 October 1992/ Accepted: 23 August 1993

\begin{abstract}
When standing human subjects are exposed to a moving visual environment, the induced postural sway forms a stable temporal relationship with the visual information. We have investigated this relationship experimentally with a new set-up in which a computer generates video images which correspond to the motion of a $3 \mathrm{D}$ environment. The suggested mean distance to a sinusoidally moving wall is varied and the temporal relationship to induced sway is analysed (1) in terms of the fluctuations of relative phase between visual and sway motion and (2) in terms of the relaxation time of relative phase as determined from the rate of recovery of the stable relative phase pattern following abrupt changes in the visual motion pattern. The two measures are found to converge to a well-defined temporal stability of the action-perception cycle. Furthermore, we show that this temporal stability is a sensitive measure of the strength of the action-perception coupling. It decreases as the distance of the visual scene from the observer increases. This fact and the increase of mean relative phase are consistent with predictions of a linear second-order system driven by the visual expansion rate. However, the amplitude of visual sway decreases little as visual distance increases, in contradiction to the predictions, and is suggestive of a process that actively generates sway. The visual expansion rate on the optic array is found to decrease strongly with visual distance. This leads to the conclusion that postural control in a moving visual environment cannot be understood simply in terms of minimization of retinal slip, and that dynamic coupling of vision into the postural control system must be taken into account.
\end{abstract}

Key words: Posture - Vision - Dynamic systems - Human

\section{Introduction}

The contribution of vision to postural stability has been a topic of research for decades (Lestienne et al. 1977;

Correspondence to: T. M. H. Dijkstra
Dichgans and Brandt 1978; Berthoz et al. 1979). In these studies it was established how the amplitude and frequency content of sway depend on the presence and nature of visual information. More recent research (van Asten et al. 1988a,b) has investigated how the temporal structure of postural sway depends on the temporal structure of visual motion. From this work we know that spontaneous sway takes place in a frequency range below about $1 \mathrm{~Hz}$ and that frequencies of visual motion must be below $0.5 \mathrm{~Hz}$ in order to induce coherent sway. The theoretical view was proposed that the postural control system can be characterized as a linear second-order low-pass system with a cutoff frequency at approximately $0.5 \mathrm{~Hz}$ (van Asten et al. 1988b).

Studying the relative timing of postural sway and motion of the visual scene is crucial if we are to understand how the postural control system is coupled to vision. Unfortunately, there are a number of technical difficulties when this action-perception relationship is to be analysed precisely. In experiments in which an actual moving room is put into motion, little control of its precise timing is possible (Lee and Lishman 1975; Stoffregen 1986). However, these stimuli do have the advantage of being realistic which, among other things, is reflected in the small amplitudes of room motion that are sufficient to induce postural sway: $3 \mathrm{~mm}$ for a viewing distance of $30 \mathrm{~cm}$ for Lee and Lishman and $2.5 \mathrm{~cm}$ for a viewing distance of $2 \mathrm{~m}$ for Stoffregen. These amplitudes are so small that they are generally not consciously perceived by the subjects. When visual scenes are generated by computer and are displayed on a screen, control over their timing is excellent. However, it is difficult to simulate realistic three-dimensional (3D) scenes. For instance, sway of an observer parallel to the screen should lead to displacements of the scene on the optic array of the observer that are larger for objects nearby and smaller for objects far away. This requires measuring the eye position of the observer (i.e. the position of the optic centre of the eye in 3D space) and a fast update of the visual scene by feedback of the eye position of the observer. Here we report on experiments in which we solved this problem 
without going way beyond perceptual thresholds. Computer displays of fronto-parallel walls were calculated in real-time based on feedback information from the measured position of the observer's eyes such as to generate displays that represent walls with a consistent depth. These walls were sinusoidally moved in a fore/aft direction in order to induce postural sway of the subject. The situation of a sinusoidally moving wall was chosen because this is interpreted easily in terms of the model that is described below. It should be noted that neither the model nor the experimental set-up is restricted to this type of stimulus. An additional advantage of this set-up over previous arrangements, which employed force plates or other apparatus to measure posture, is that it gives the position of the eye in 3D space. This allows us to calculate precisely the optic flow on the optic array of the subjects caused by head movements. In the experiment, we varied the mean distance between subject and wall as the experimental variable. Distance is known to play an important role in postural control (Lee and Lishman 1975; Paulus et al. 1989). Furthermore, in the model put forward by Schöner (1991), distance determines the stability of the action-perception cycle, contrary to e.g. the frequency or the amplitude of the movement of the stimulus which have no influence on stability.

With this set-up we address a number of questions about the temporal relationship of postural sway and wall movement in the fore/aft direction that are posed by a recent model on the dynamics of this action-perception pattern (Schöner 1991). This model characterizes the postural state controlled by the nervous system in terms of the position of the eye in the physical environment. Many sensory processes other than vision may contribute to determine this postural state, such as proprioception, vestibular information and pressure sensing in the foot. The idea is that all of these sensory processes contribute to a dynamical system such that the equilibrium postural state (upright posture) is an attractor solution. It is important to note that this dynamical system is not the same as the physical system of the passive biomechanical system involved in posture. Instead, the control properties of the nervous system including reflex loops and active control systems are cast into the form of a dynamical system. The visual influence on postural control is parametrized by the expansion rate of the fixated object on the retina. This expansion rate is formally equal to the inverse of "time-to-contact" independently of object size and distance (Lee 1980; Tresilian 1991). The parametrization of visual influence by the expansion rate is not essential: other parametrizations, like the spatial mean of the optic flow, will have the same temporal characteristics and lead to the same model predictions. They only result in a rescaling of the coupling constant, which captures the strength of the visual influence on the postural control system (see below).

In the model the assumption was made that the nonvisual contributions can be represented by a linear, second-order dynamical system. Additive coupling (via a coupling constant) to the expansion rate leads to a linear driven oscillator. When the visual surround is oscillating, the model predicts that vision would make two contribu- tions to the dynamics. Firstly, it would stabilize posture by enlarging the effective damping. The size of this extra stability would depend on the distance to the wall and on the coupling constant; stability would decrease with increasing distance. Secondly, according to this model, vision drives the dynamics with the frequency of the oscillation. The effective amplitude of the drive is the amplitude of the movement of the visual surround divided by the mean distance to the surround. It is important to note that this model tends to minimize expansion rate, but does not always do so, depending on the frequency of the visual drive and on the stability. Driving frequencies that are very different from the eigenfrequency lead to large phase delays and thus to large expansion rates. Low stability leads to more variability in phase delay and thus to larger mean expansion rates. The hypothesis of minimization of retinal slip has been put forward to explain the observed increase of RMS amplitude of postural movements when the distance to a static surround is increased (Lee and Lishman 1975; Paulus et al. 1989). These last authors assume a threshold for detection of retinal slip and assume that the postural control system minimizes the supra-threshold retinal slip. The model is consistent with this increase in RMS because the stability in the model decreases with increasing distance. The model can be regarded as an extension of the minimization hypothesis to a dynamical context. The main difference between the hypothesis of retinal slip minimization and the model is that the latter explicitly models the intrinsic dynamics of the postural control system.

The experimental results obtained by van Asten et al. $(1988 a, b)$ and by Berthoz et al. (1979) are compatible with the model, and a related model had already been proposed by these authors. The formulation given by Schöner (1991) stresses the temporal stability of the action-perception pattern. Stability is postulated to underlie both the persistence of phase locking between stimulus and response in the face of fluctuations as well as the return to phase-locked behaviour following an external perturbation. This postulate and a number of other predictions cannot be tested on the basis of these older data, because methods to measure the stability of the relative timing of stimulus and sway were not implemented, and no perturbations of that relative timing were performed. Here we calculate two measures of temporal stability from the data: (1) the variance of relative phase, evaluated from a time series of relative phase and (2) the relaxation time of the action-perception pattern obtained by determining the time it takes the system to recover its stable relative timing pattern after an abrupt phase shift of the sinusoidally moving visual array. We manipulate the temporal stability by varying the distance between the eye and the visual scene. We have tested the concrete model predictions that as visual distance increases, (1) temporal stability decreases; (2) the time delay between visual drive and postural response increases (when the eigenfrequency of sway control is lower than the driving frequency); (3) the amplitude of postural sway decreases; (4) the expansion rate of the visual surround on the optic array of the observer decreases. 


\section{Experimental set-up}

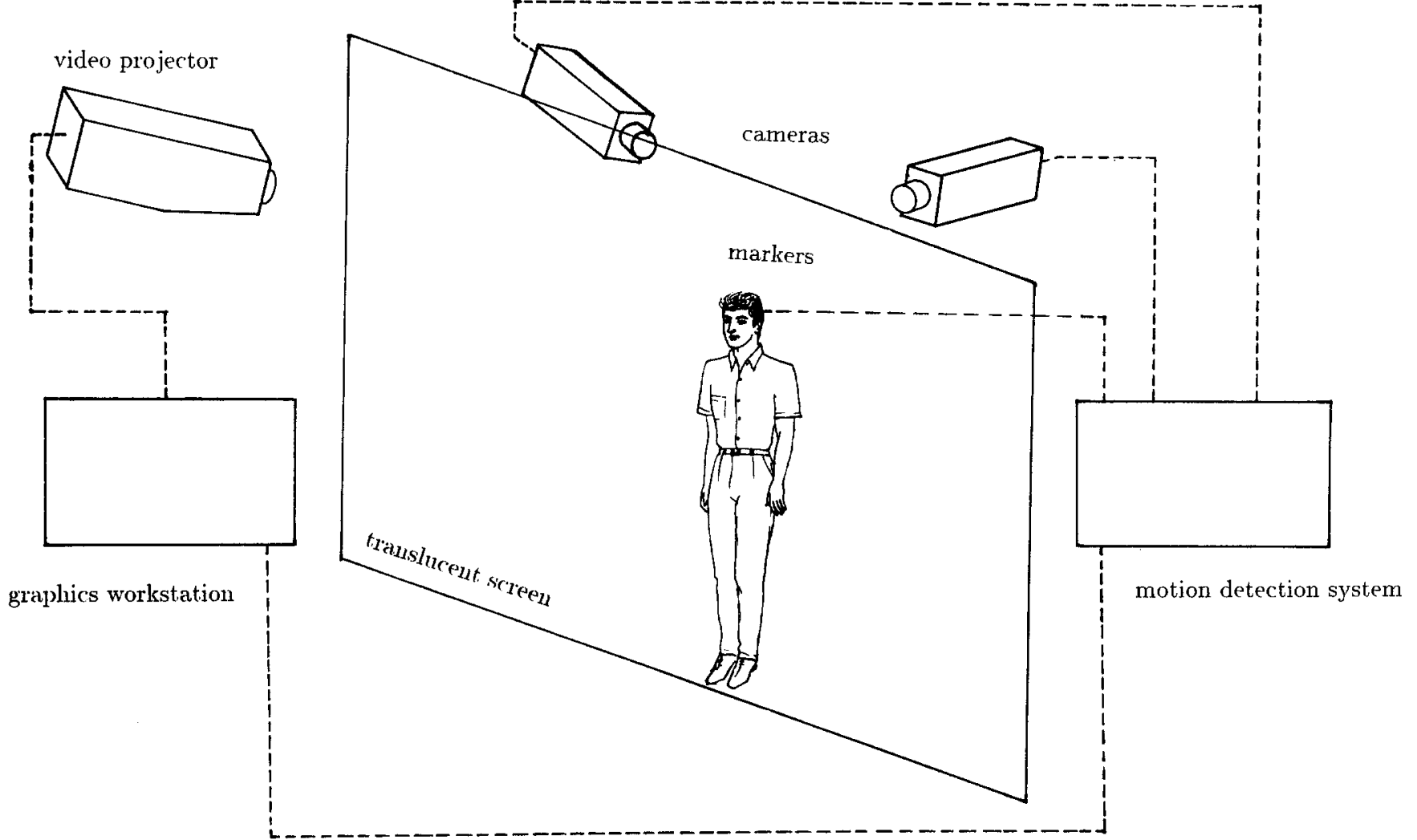

Fig. 1. Experimental set-up

\section{Materials and methods}

\section{Experimental set-up}

Red/green stereograms were generated by a SUN4/260 CXP workstation running under SunOS 4.0.3 and were projected onto a translucent screen of dimensions 2.5 by $2 \mathrm{~m}$ by a Barco Graphics 400 video projector (red phosphor p56, green phosphor p53). The green stimuli were barely visible through the red filter (Kodak Wratten 25), transmission being less than $2 \%$, while the red stimuli were invisible through the green filter (Kodak Wratten 58), transmission being less than $0.5 \%$. The screen was homogeneously white without any visible texture. The subject wore a pair of goggles that contained the filters and limited the field of view to approximately $120 \mathrm{deg}$ wide by $100 \mathrm{deg}$ high. Due to the restriction of the viewing range, the edge of the screen was not visible to the subject.

The subject stood approximately $50 \mathrm{~cm}$ in front of the screen wearing a flat-topped helmet on which six diodes emitting infrared light (ireds) were mounted (Fig. 1). The positions of these markers were measured with two cameras of a Watsmart system (Northern Digital ) at a rate of $400 \mathrm{~Hz}$. The two cameras were placed approximately $2 \mathrm{~m}$ behind and $1.5 \mathrm{~m}$ above the subject in order to have the best possible signal-to-noise ratio for detecting movement in a horizontal plane. The walls and ceiling of the room were covered with infrared-absorbing cloth. This same cloth also hung in front of the screen leaving only a small, door-sized window for the subject to stand. With these precautions we never encountered difficulties with reflections of the infrared light.

The 2D coordinates of the two cameras were converted realtime into 3D coordinates and sent to the SUN4. This computer was programmed to generate a new stereogram of the wall from the current viewpoint of each eye of the observer, using an algorithm to be explained below. With this set-up, every frame $(15 \mathrm{~ms})$ provided a new view of the simulated wall. The mean $3 \mathrm{D}$ position of the viewpoints, the position of the cyclopean eye, and the orientation of the head were stored, together with the position of the stimulus, for later analysis. These signals were sampled at a rate of $66 \mathrm{~Hz}$. The delay in the feedback loop between eye translation and position change of a pixel in the middle of the screen was measured using a turntable and found to be $43 \pm 3 \mathrm{~ms}$. The small variability was probably caused by the fact that SunOS is not a real-time operating system.

The position of each eye in 3D space was calculated using a quaternion algorithm described by Horn (1987). Each session started with a calibration in which the subject faced the cameras and held two additional markers in front of the eyes. The position of all ireds in this configuration was sampled for $200 \mathrm{~ms}$ at $200 \mathrm{~Hz}$, and from these data the position of each eye relative to each marker of the helmet was calculated. Then the experiment would start with the subject facing the screen. The rotation of the helmet relative to the orientation in the calibration procedure was calculated using a real-time implementation of Horn's algorithm for a planar figure (Sect 5 of Horn 1987). Thus the position of each eye could be calculated during the experiment using the positions of the eyes with respect to the ireds on the helmet. It should be noted that we do not measure the orientation of the eyes relative to the head (rotations of the eyes in their sockets) but the position of each eye in 3D space. Assuming the accuracy of the Watsmart system to scale linearly with the calibrated volume, which in our case was a cube with sides of length $0.6 \mathrm{~m}$, we estimate the accuracy for the position of one marker to be $3 \mathrm{~mm}$ (Ball and Pierowsky 1987). From this we estimate the systematic error of eye position relative to the simulated wall to be of the order of $1 \mathrm{~cm}$. The dynamic noise in the eye position is approximately white and has a standard deviation of $1 \mathrm{~mm}$. 


\section{Stimuli}

The stimuli simulated a fronto-parallel wall covered with 140 stereo dots, each with a size of $0.2 \mathrm{deg}$ by $0.2 \mathrm{deg}$. The simulation was implemented by keeping a list of the dots in the memory of the graphics processor. The projection matrices (one for each eye) were recalculated every frame on the basis of the most recent positions of each eye and on the sinusoidal translation of stimulus. The density of dots was uniform per solid angle as seen from the view position when the experiment started. Therefore, dot density could not provide a cue to the distance to the wall. The dots lay in an annulus between $10 \mathrm{deg}$ and $60 \mathrm{deg}$ visual eccentricity and thus had a density of $0.056 \%$. The hole in the middle of the stimulus was made to suppress the visibility of aliasing effects, which are most visible in the foveal region. The spatial resolution of the system was 1152 by 900 pixels and the frame rate was $66 \mathrm{~Hz}$.

The wall was suggested to be at distances of 25,50,100 and $200 \mathrm{~cm}$. The first is suggested to be in front of the screen, the second on the screen and the last two behind the screen. Distance was suggested both by stereo vision as well as by simulation of the geometrically correct displacement of the image on the screen, using feedback of the eye position of the observer. Subjects reported that stereo vision strongly enhanced the perceptual quality of the display and made viewing for longer times more comfortable. However, it is not essential for our experiment, since in a pilot experiment to compare trials with and without stereo vision we found no significant differences. Subjects might also use accommodation as a distance cue but this effect has been shown to be very weak (Fisher and Ciufredda 1988).

The wall was sinusoidally driven with an amplitude of $4 \mathrm{~cm}$ and a frequency of $0.2 \mathrm{~Hz}$. Each of the four distances was measured both with and without perturbations, giving a total of eight conditions. The perturbed trials had three perturbations of $180 \mathrm{deg}$ each, always at the point of maximum velocity (so there was no discontinuity in position, cf. Fig. 2) and occurring at random moments. Each condition was repeated four times, and the order of trials was random. The experiment was conducted in two sessions of approximately $1 \mathrm{~h}$ each and always started with a trial without any visual stimulus for dark adaptation of the subject. In all there were 34 trials for each subject. Each trial lasted for $140 \mathrm{~s}$; the first $20 \mathrm{~s}$ were for adaptation and were not stored.

\section{Analysis of data}

Data were analysed in two ways, by a linear time-invariant analysis, which is quite common in physiology (Marmarelis and Marmarelis 1978), and by a dynamic approach, as described, for example, by Schöner and Kelso (1988). This approach was used in the context of postural control by Schuster and Talbot (1980). The data for both types of analysis were the sinusoidal motion of the wall and the response of the subject in the fore/aft direction sampled at $66 \mathrm{~Hz}$ for 2 min. Both types of analysis were implemented in MATLAB version 3.5 i on a SUN4.

In the linear time-invariant analysis we calculated the Fourier transform of the drive and the response of the subject. From these the spectra of the magnitude squared coherence (MSC), the phase and the gain were calculated. The MSC is a measure for the strength of locking of the subject to the movement of the wall if the system is linear, or of the relative contribution of the linear part of the system if the system has non-linear components (Carter 1987). All spectra were only evaluated at the driving frequency because there were never any peaks at other frequencies in the spectra. All spectra were calculated with a Welch procedure (Marple 1987) in order to obtain unbiased estimates of the spectra. For the unperturbed trials we used 15 overlapping segments each $15 \mathrm{~s}$ long and a factor 7 zero padding. The MSC depends strongly, the phase and gain very weakly on the number of segments (Carter 1987). We only use the variation of the MSC with distance and this is independent of the number of segments. For the perturbed trials we excluded two cy- cles after a perturbation to assure stationarity and for each of the remaining four parts we used seven segments and a factor 3 zero padding to get approximately the same segment length as in the unperturbed case. We always scaled the Fourier-transformed signals in such a way that Parseval's theorem would hold, i.e. that the power in the original signal and in the Fourier-transformed signal is the same.

Fouriertechniques were also used to determine the amplitude of the expansion rate of the stimulus on the optic array of the subject (the inverse of time-to-contact). We first calculated a time series of the retinal eccentricity of the edge of the stimulus $\theta$ at a distance $Z(t)$ by $\tan \theta=X_{o} / Z(t)$, with $X_{o}$ the distance between the centre and edge of the stimulus (physical size). From this we extracted a time series of expansion rate by dividing the time derivative of $\theta$ by $\theta$. In the terminology of Tresilian (1991) this would be the inverse of dilatation tau, denoted by $\tau_{1}^{(1)}$. This time series of expansion rate, being constructed from a difference of two sinusoidal signals (i.e. the motion of the wall and of the subject) is itself a sinusoidal signal at the same frequency as the drive. As a rough characterization of the time series of expansion rate we calculated its amplitude by Fouriertransforming it and taking the height of the peak in the spectrum, which always occurred at the driving frequency, as the amplitude of the expansion rate.

The dynamic analysis amounted to calculating a discrete time series of relative phase (phase of stimulus minus phase of response; this definition entails a different sign convention than, for instance, that of van Asten 1988a) as follows. From the input and response data the significant extrema both of position and velocity traces were picked using a peakpicker. Before peakpicking, the data were smoothed using a gaussian window with a standard deviation of $0.25 \mathrm{~s}$. The criterion for significance of an extremum was a fraction of the range (i.e. the difference between maximum and minimum) in a segment of $5 \mathrm{~s}$ before and $5 \mathrm{~s}$ after the extremum. An extremum in position was accepted as significant when it differed more than $40 \%$ from the neighbouring extrema. For an extremum in velocity the criterion was $70 \%$. We chose these different percentages because velocity tended to be somewhat more peaked than position. The results depend weakly on the precise values of these percentages. From the eight time series of extrema (maxima and minima, both position and velocity of both input and response), relative phase was calculated with the drive as reference and the response as target. This was done by matching each extremum in the reference signal to all extrema in the target signal of the same type and within half a cycle before and half a cycle after the extremum. A relative phase value was calculated by taking the time difference between two extrema of the same type and dividing this by the time difference between two extrema in the reference signal. As the time value of this relative phase we used the time of the target extremum. The result of these manipulations are four time series of relative phase: maxima and minima of position and velocity. Because these four time series were not very different (see Results) we combined these time series in one overall time series of relative phase (see Fig. 2 b,d). As we have four samples per cycle the mean sampling frequency is $0.8 \mathrm{~Hz}$.

For all trials we calculated mean phase and angular deviation (Batschelet 1981) from the combined time series of relative phase, using circular statistics. Mean phase is a measure of the time delay between input and response, and angular deviation is a measure of the stability of the response. For the perturbed trials we excluded the data points in the two cycles after the perturbation, because mean phase and angular deviation are measured for the stationary behaviour. The relaxation time, the time it takes the system to regain its in-phase behaviour after a perturbation, was estimated as follows. We defined a band around the mean of relative phase of the unperturbed part of 1.5 times its angular deviation. After a perturbation the phase generally leaves this band and we calculated relaxation time by fitting an exponential to the points outside the band and the first four points inside the band (cf. Fig. 2). The fit was implemented by linear regression on the logarithm of the absolute phase values. As a rough quality measure of the fit we took the explained variance of the fit divided by the angular deviation. If the 
relative phase did not leave the band or if the quality measure was lower than 2, we excluded the perturbation. The relaxation time of a trial was the average of the non-excluded relaxation times in a trial. This relaxation time depends somewhat on the parameters used, but the trend with distance does not depend on them. It should be noted that this procedure probably somewhat underestimates the larger relaxation times: these generally occur for lower stability, which means that the band is wider so the system is bound to return faster inside the band.

\section{Subjects}

Four subjects with normal vision or vision corrected to normal and normal stereo vision were tested in all conditions. Three of the subjects were familiar with the purpose of the experiment. The main findings were confirmed by a fifth subject who was naive as to the purpose of the experiment. This subject was not tested in all conditions of the experiment. Subjects were instructed to look at the centre of the stimulus and to stand relaxed; they stood on a firm stable support in normal Romberg posture.

\section{Results}

Results were obtained for four subjects whose order of presentation will be: G.S., M.G., S.G., C.K. Unless otherwise noted, all statistical tests are one-way ANOVAs with distance as the independent variable at a significance level of $5 \%$. For post hoc analysis (pairwise comparison) we used the Newman-Keuls procedure (Hays 1988), also at a significance level of 5\%. In order to keep the burden down we averaged over subjects, which is allowed because the assumption of sphericity was never violated (Hays 1988).

Generally the subjects responded to the sinusoidally moving wall with an almost sinusoidal postural response in the fore/aft direction. They did not consciously perceive visual motion, except for the condition where the wall was nearest $(25 \mathrm{~cm})$ and at the perturbations. Those parts of the trials where the response did not show a clear sinusoidal pattern were excluded from the analysis. For the four subjects the proportion of data excluded was $7 \%, 22 \%, 0 \%$ and $2 \%$, respectively. Most of these exclusions occurred in the first few trials after the start of the experiment, or after the midway break, and for a suggested distance of 100 or $200 \mathrm{~cm}$. We interpret the fact that the trials with non-oscillatory parts occurred primarily subsequent to a break as a sign of long term adaptation. The detailed study of such adaptation effects is, however, beyond the scope of this contribution. In relation to the linear dynamic model, we note that a decreased oscillatory response component is expected at larger distances: stability is so low that phase-locked behaviour sometimes does not occur.

As a descriptive measure for our data we calculated the RMS of the position and velocity signals of subject movement in the fore/aft direction. Neither of these changed significantly with distance for any of the subjects. Averaged over all subjects the RMS of the position signal at a distance of $200 \mathrm{~cm}$ decreased by $13 \%$ relative to the RMS at $25 \mathrm{~cm}$. For the velocity the decrease was only $2 \%$. The other signals (left/right and up/down trans- lation of the eye and orientation of the head) showed little variation. The left/right translation was by far the largest with a RMS of approximately $8 \mathrm{~mm}$.

The means and angular deviations of the four time series of relative phase were generally significantly different as revealed by two-way ANOVAs with distance and type (minima or maxima of position or velocity) as independent factors. For an example of this difference see Fig. $2 \mathrm{~b}$, where the estimates of relative phase after the perturbation based on velocity are larger than the estimates based on position. The differences caused by type were not consistent across subjects except that the angular deviation of the relative phase calculated from the velocity signals was higher. This is to be expected since velocity is derived from the position by differentiation, a noise-enhancing procedure. The ANOVAs revealed no interaction between type and distance, and as we are interested in the effect of distance we combined the four time series. Further, after a perturbation the combined time series of relative phase generally is nicely approximated by an exponential (Fig. 2), indicating that it is reasonable to combine the individual time series.

All statistical parameters calculated from the stationary part of the perturbed trials and from the unperturbed trials were never significantly different, so the perturbations did not change the stationary behaviour. Therefore we only show the results of the perturbed trials.

\section{Stability}

In Fig. 3-5 three different measures for the temporal stability are plotted. All three indicate a decrease of stability with increasing distance. The MSC (Fig. 3) decreases with increasing distance. This decrease is significant for subjects G.S. and S.G. The mean decrease over all subjects between $25 \mathrm{~cm}$ and $200 \mathrm{~cm}$ is approximately $10 \%$. Post hoc analysis revealed all pairs of conditions to be significantly different except the $25 / 50 \mathrm{~cm}$ pair.

The increase in angular deviation of relative phase (Fig. 4) is significant for three subjects, but not for subject S.G. Also note that the stability across subjects as reflected in the angular deviation shows almost exactly the same pattern as reflected in the MSC: subjects S.G. and C.K. are strongly phase-locked to the stimulus, G.S. is in the middle and subject MG shows the weakest phase locking. This is also reflected in the correlation between MSC and angular deviation: $-0.75,-0.84,-0.78$ and -0.50 , respectively. The mean increase over all subjects in angular deviation from $25 \mathrm{~cm}$ to $200 \mathrm{~cm}$ is approximately $45 \%$. Post hoc analysis showed only the $200-\mathrm{cm}$ condition to be significantly different from all others.

The relaxation after a perturbation generally shows a clear exponential decay in phase. Figure 2 shows two data records and the derived time series of relative phase. Figure $2 \mathrm{a}$ is from a trial with a small distance $(25 \mathrm{~cm})$ between subject and wall and shows a large stability as reflected in the fast relaxation after the perturbation and in the small angular deviation. Figure $2 \mathrm{c}$ is from a trial with a large distance $(200 \mathrm{~cm})$ between subject and wall and shows little stability as reflected in the slow relax- 

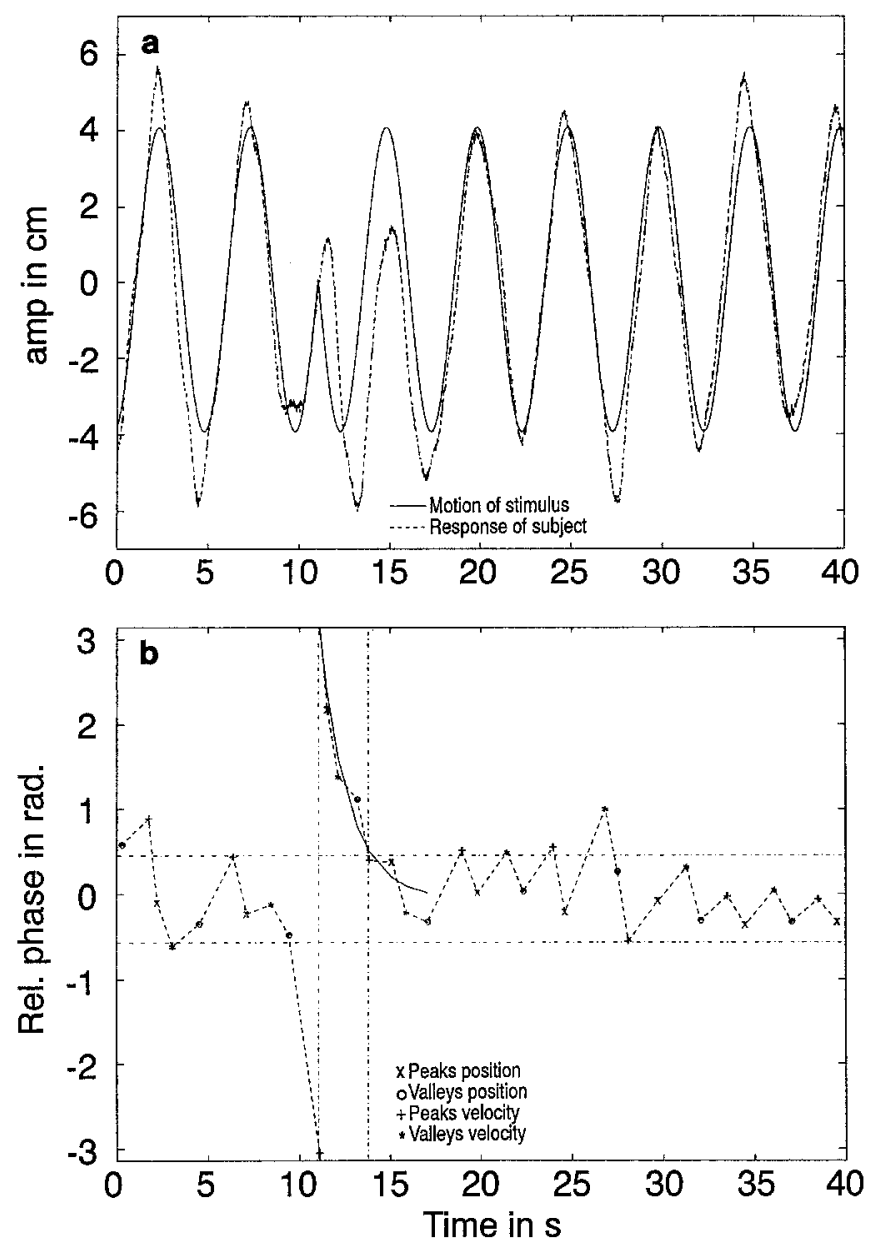

Fig. 2A-D. Examples of data records and the derived time series of relative phase. a Position traces of visual drive (solid line) and postural response (broken line) for a small mean distance $(25 \mathrm{~cm})$ between subject and wall. b Derived time series of relative phase (broken line) with estimation of relaxation time (solid line). Symbols at the lower left refer to the different types of phase values. Also given are the time of the perturbation (left vertical line) and the time when
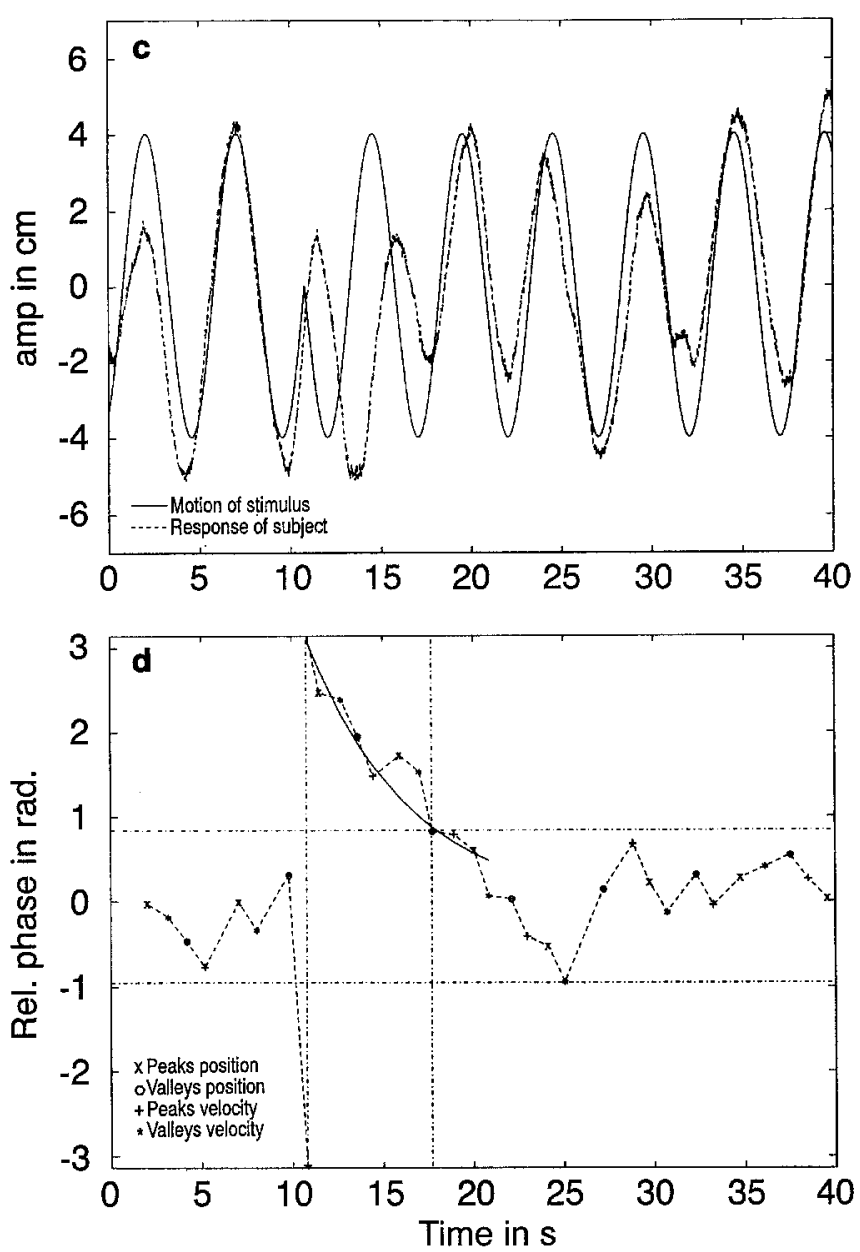

the relative phase enters the band around the mean phase (right vertical line). The two horizontal lines are plus and minus 1.5 times the angular deviation around the mean. $\mathbf{c}$ Position traces of visual drive and postural response for a large mean distance $(200 \mathrm{~cm})$ between subject and wall. d Derived time series of relative phase with estimation of relaxation time

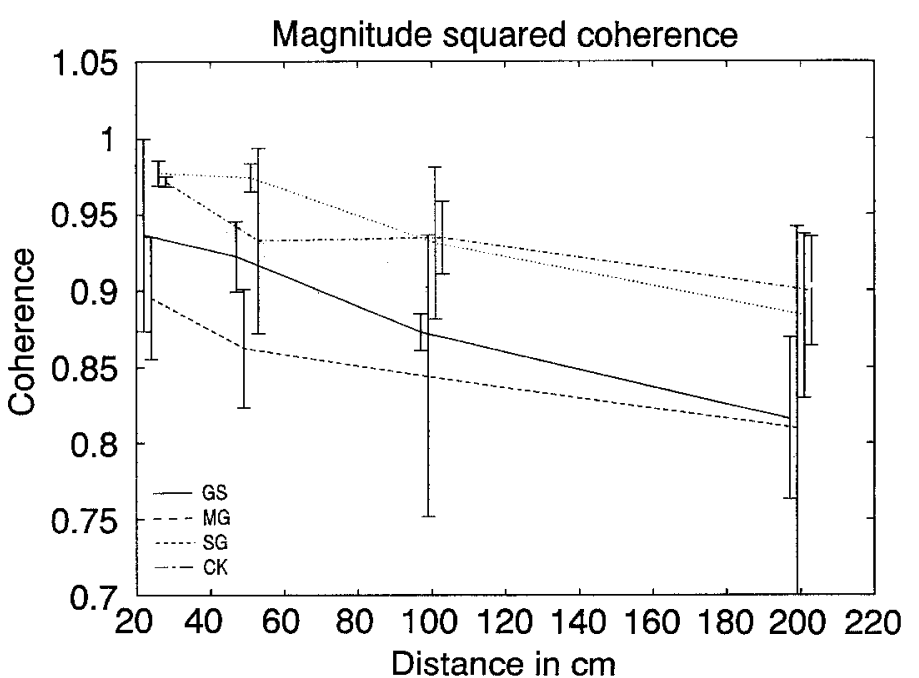

Fig. 3. The magnitude squared coherence as a function of distance. The line style for each subject is shown at the lower left and is the same in subsequent plots. The vertical bars denote the standard deviation and are slightly displaced horizontally for clarity

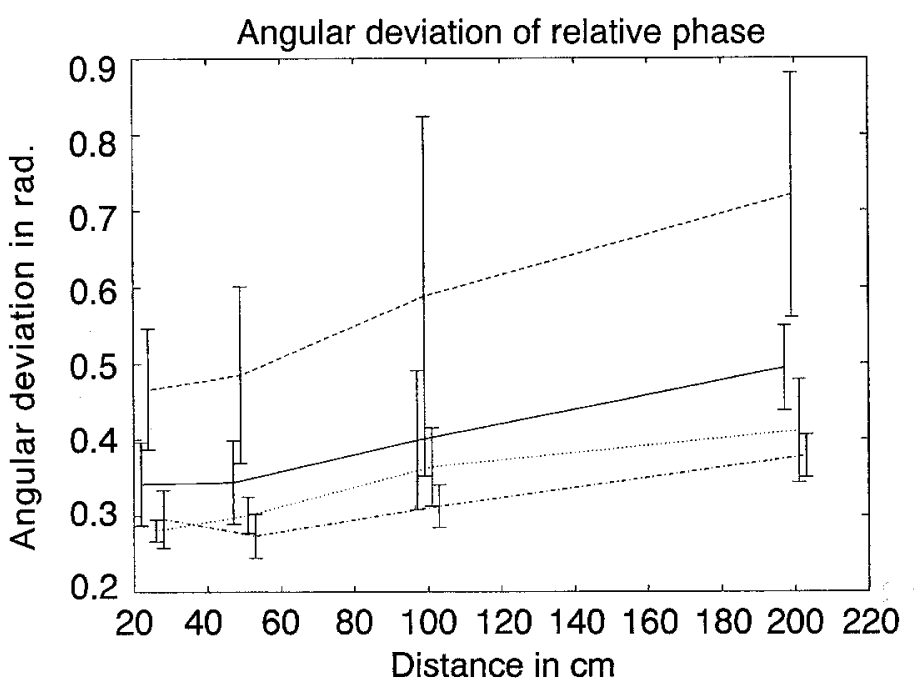

Fig. 4. The angular deviation of relative phase as a function of distance. For details see legend of Fig. 3 


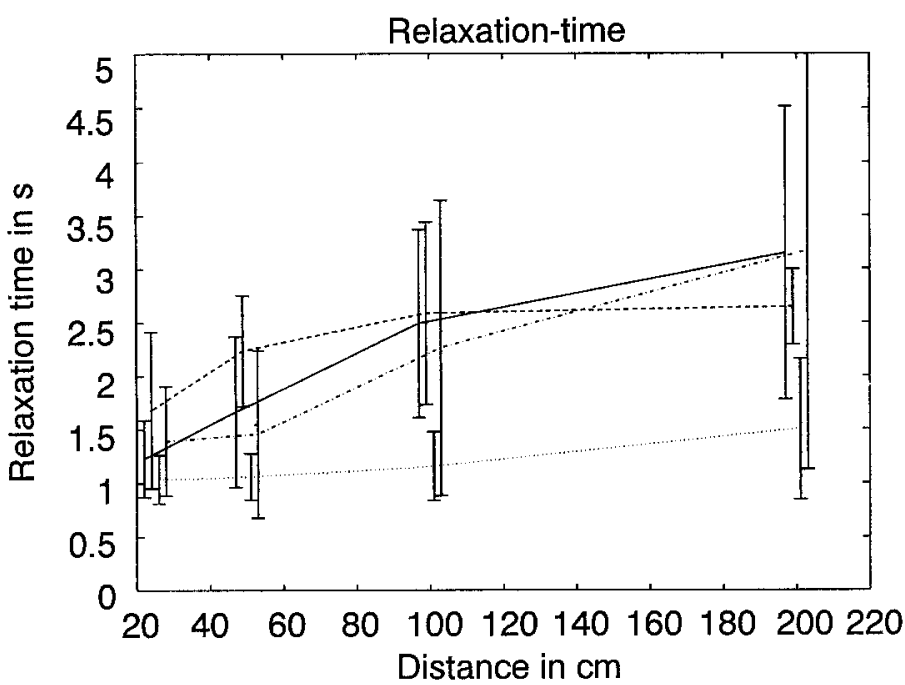

Fig. 5. The relaxation time as a function of distance. For details see legend of Fig. 3

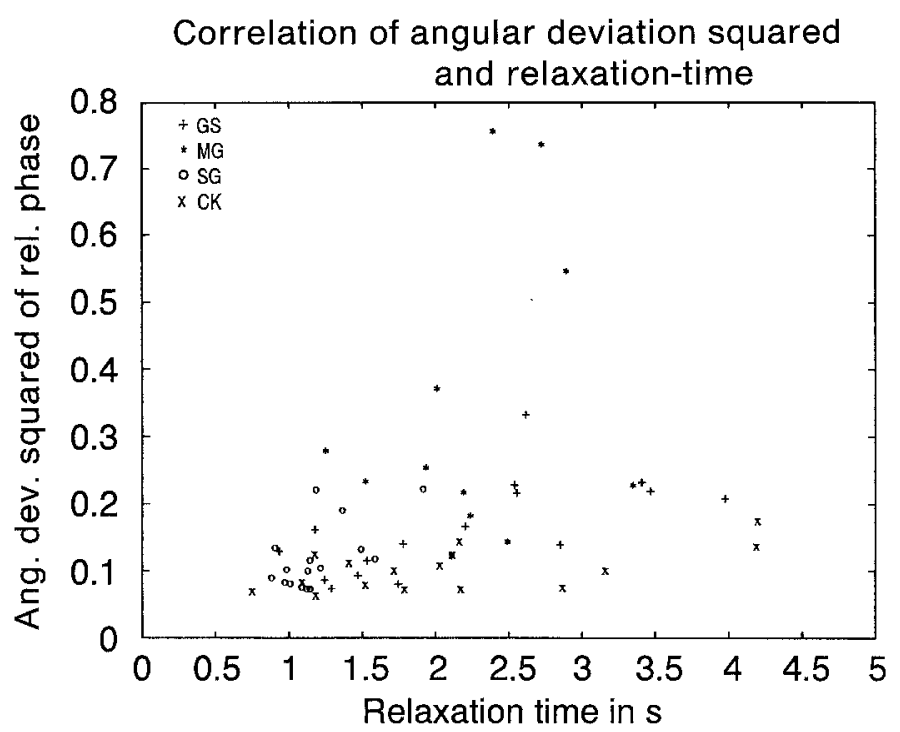

Fig. 6. Scatter plot of relaxation time and squared angular deviation of relative phase

ation after the perturbation and in the large angular deviation. Those cases that did not show this exponential decay were excluded. For the four subjects, the proportion of perturbations excluded was $17 \%, 44 \%, 6 \%$ and $6 \%$, respectively. Roughly half of these exclusions were caused by non-oscillation, as previously discussed. The remaining exclusions almost always occurred for distances of 100 and $200 \mathrm{~cm}$, where stability is lowest. The exclusion was usually caused by the fact that it took the subjects a very long time (longer than $15 \mathrm{~s}$ ) to return to in-phase behaviour. During this period the phase would drift considerably, causing our exponential fit to be a very bad approximation. The increase in relaxation time (Fig. 5) is significant for three subjects, but not for M.G. The mean increase over all subjects from $25 \mathrm{~cm}$ to $200 \mathrm{~cm}$ is approximately a factor 2 . A separate ANOVA to test for an effect of the order of the perturbation within a trial revealed no effect of order on relaxation time. This is consistent with the fact that we never found any significant difference in the statistics between perturbed and unperturbed trials. Post hoc analysis revealed all pairs of conditions to be significantly different except the 25 / $50 \mathrm{~cm}$ pair.

The theory predicts a covariance between angular deviation squared and relaxation time. Figure 6 gives a graphical impression of this covariance. The correlation coefficients for the four subjects are 0.67, 0.29, 0.61 and 0.62 , respectively. Only the coefficient of subject M.G. is not significantly different from zero. This is probably caused by the small number of included relaxations at a distance of $2 \mathrm{~m}$ ( 2 out of 12). The temporal stability of this subject at this distance is so low that he often failed to return to a stable in-phase pattern after a perturbation within a few cycles. This of course biases the correlation, because we only take the fast relaxations.

\section{Delay}

In Fig. 7 two different measures for the time delay between visual drive and postural response are plotted. In Fig. 7a the delay as calculated from the phase spectrum is plotted, whereas in Fig. $7 \mathrm{~b}$ the mean phase difference as calculated from the time series of relative phase is shown (note that our definition of delay entails a sign convention different from that of, for example, van Asten 1988a).

Both indicate an increase of delay with increasing distance. There is a significant effect of distance on both measures for three subjects, not for M.G. The correlation between delay as calculated from the phase spectrum and as calculated from the time series of relative phase are large: $0.93,0.97,0.99$ and 0.98 , respectively. This indicates that the procedure we used to calculate relative phase leads to sound results. Post hoc analysis showed all pairs of conditions to be significantly different except the 25 / $50 \mathrm{~cm}$ pair and the $100 / 200 \mathrm{~cm}$ pair.

\section{Gain and optic flow}

In Fig. 8 the gain is plotted as a function of distance. It shows a slight decrease which is significant only for subject S.G. Further, the gain is near 1, which means that the amplitude of the response of the subjects is the same as the amplitude of the drive. Especially for the smaller distances, the gain is even larger than 1 , indicating overcompensation for the perceived ego-motion. Post hoc analysis showed that only the $200-\mathrm{cm}$ condition was significantly different from all others.

Figure 9 shows the amplitude of the expansion rate of the visual surround on the optic array of the subject. This expansion rate is calculated using the sampled movements of the subjects relative to the movements of the wall and therefore depends on the distance to the wall and on the gain and the delay of the response. For reference, we also include a curve which gives the expansion rate if the subject did not move. The expansion rate decreases significantly for all subjects. Note that while the 

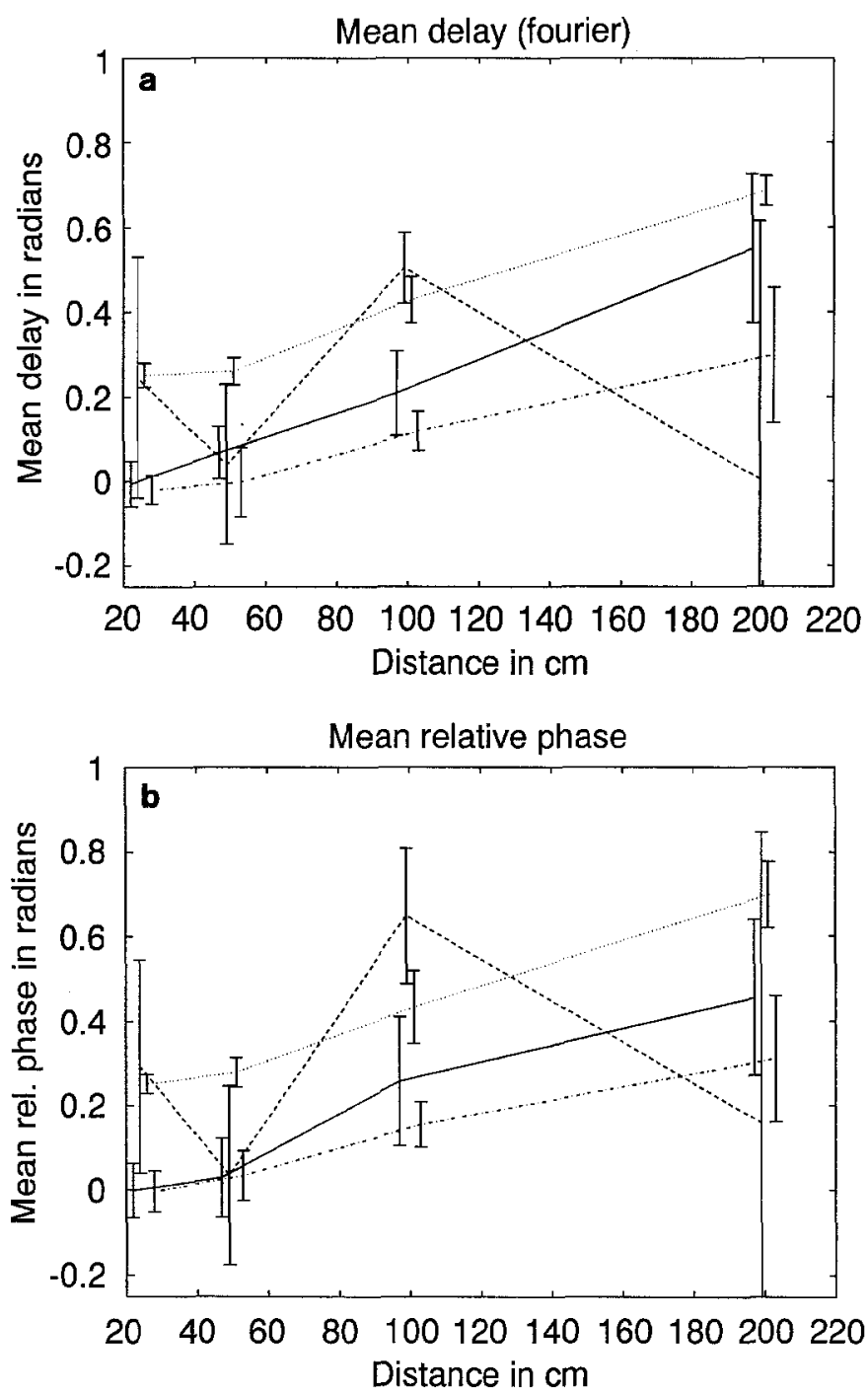

Fig. 7A,B. Delay as a function of distance. a As calculated by Fourier analysis. $\mathbf{b}$ As calculated from the mean of the relative phase time series. For details see legend of Fig. 3

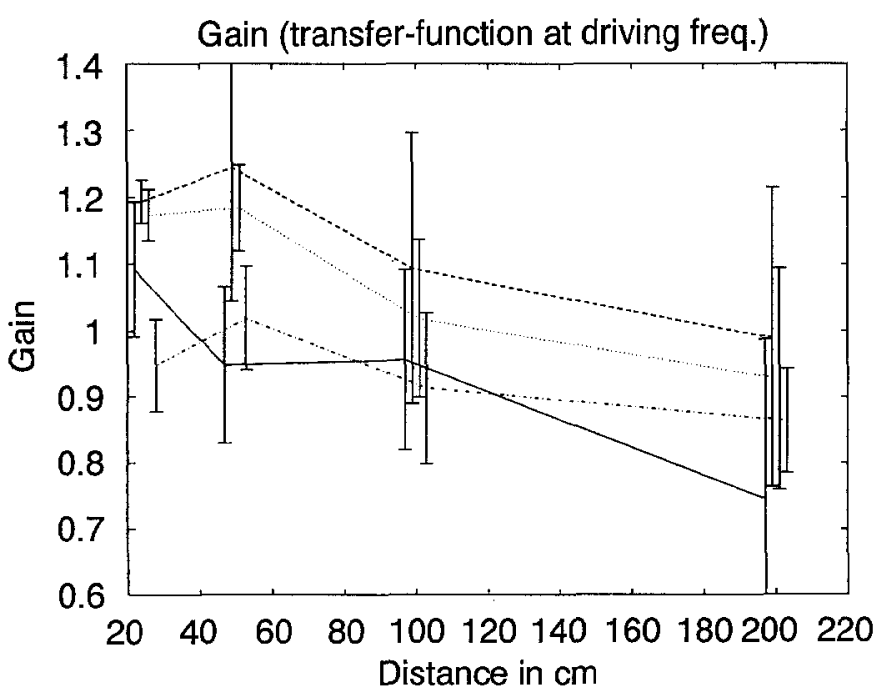

Fig. 8. Gain as a function of distance. For details see legend of Fig. 3

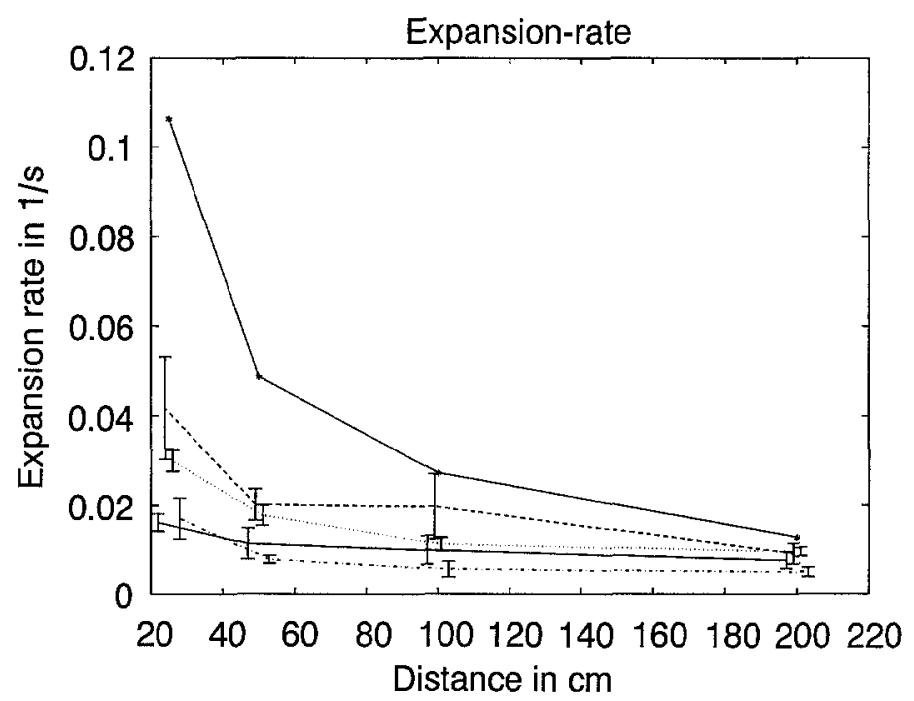

Fig. 9. Strength of the expansion rate of the visual surround on the optic array of the observer as a function of distance. The upper curve gives this amplitude if the subject does not move. For details see legend of Fig. 3

gain decreases by approximately $20 \%$ with increasing distance, the expansion rate decreases by approximately $70 \%$. Post hoc analysis revealed that all conditions were significantly different from one another.

\section{Discussion}

This investigation involved experiments on visually induced postural sway in which information from the measured position of the eyes is fed back to the display computer in such a way that it generates scenes with realistic geometry and consistent depth. Our focus was on the temporal relationship between stimulus movement and postural response. To characterize this relationship we measured the relative phase between sway movement and the sinusoidal motion of the visual scene. This provides us with a time series characterizing the temporal evolution of this relationship. We manipulated visual distance because this parameter is predicted to affect the temporal stability of the action-perception cycle (Schöner 1991).

We found an enhanced variability of the timing of sway relative to the sinusoidal motion of the visual surround as the distance to the wall increased. Further we found a larger relaxation time after a phase perturbation of the sinusoidal motion of the visual surround at larger distances. The correlation between the two measures was about 0.6 . This shows that processes underlying recovery from perturbations and the processes controlling fluctuations of the unperturbed action-perception cycle are governed by the same temporal stability. In the model of Schöner there is an exact relation between angular deviation and relaxation time. Because the systematic change in temporal stability induced by our distance manipulation is not very large compared to the fluctuations in the angular deviation and relaxation time (see the large error 
bars in Figs. 4,5), we cannot, on statistical grounds, expect a large correlation between these two stability measures. Beyond statistics, we have no firm explanation for this low, although significant, correlation. In a separate analysis we ruled out one possible explanation by finding no significant effect when we tested whether the phase value just prior to the perturbation might influence the relaxation time. An explanation that remains possible is the fact, already mentioned, that the procedure to calculate the relaxation time may well underestimate relaxation time for higher values of the angular deviation. It should be noted that our analysis of relaxation presumes the existence of a stable attractor (in-phase behaviour). When this attractor is very weak, as in the case of the excluded perturbations, one might expect the relaxation to be strongly influenced by noise. Thus the fact that we had to exclude some of the relaxations when stability is low, is qualitatively in agreement with the model. Generally, such exclusions are conservative with respect to the hypothesized decrease in stability.

The decrease of stability is also reflected in the MSC, although somewhat less sensitively. The observed decrease of temporal stability as visual distance increases is consistent with the predictions of the model. In the model, this decrease is caused by the decrease of the amplitude of visual expansion rate (also experimentally observed, see Fig. 9), which leads to a reduced effective coupling strength of posture to the time structure of visual motion.

Secondly, we showed an increase of the delay between sway and visual motion as visual distance is increased. This observation is likewise consistent with the model. In the model, this effect leads to the hypothesis that the eigenfrequency of the sway control system is lower than the driving frequency for all subjects. Eigenfrequencies of $0.2-0.15 \mathrm{~Hz}$ have been reported before (van Asten et al. 1988a). Surprising here is the small range of eigenfrequencies that is compatible with the data: eigenfrequencies larger than $0.2 \mathrm{~Hz}$ lead to a system that has a phaselead relative to the drive, and eigenfrequencies below 0.15 lead to a steep increase in delay. Only the small range of 0.16 to $0.19 \mathrm{~Hz}$ leads to reasonable results. So despite the considerable difference in biomechanics between the subjects, the eigenfrequency is strongly constrained in this experiment.

Thirdly, there was no significant change of the amplitude of postural sway as visual distance was varied. Sway amplitude always closely matched the amplitude of the visual motion. We have found this result before (Dijkstra et al. 1992). The slight, non-significant decrease of the gain is not by any means as strong as that predicted by the model and therefore is quantitatively in contradiction with the model. From an ecological viewpoint, however, this result makes sense: subjects correct their posture by matching the amplitude of their egomotion with the visual motion, irrespective of the distance to the visual surround.

Finally, we found that the expansion rate of the visual surround on the optic array of the observer was not constant. This contradicts the hypothesis that the system controls posture purely by minimizing the expansion rate
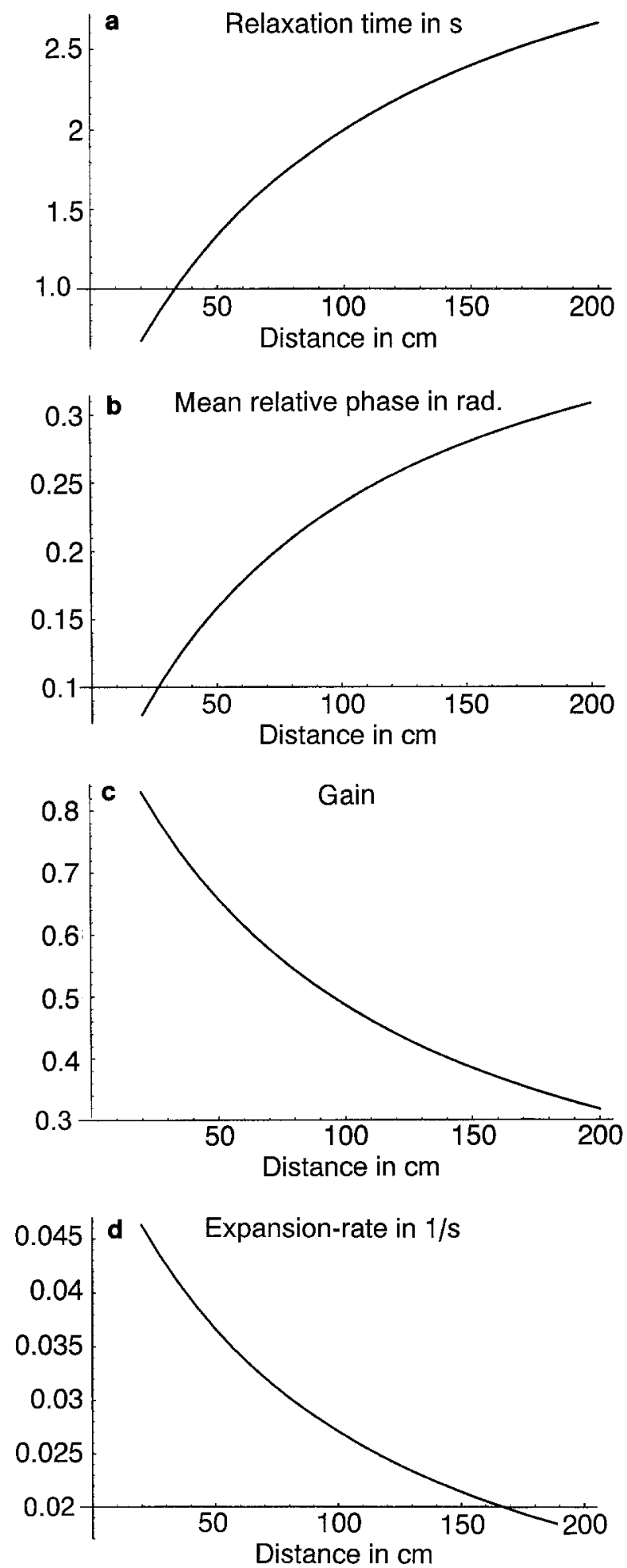

Fig. 10. Predictions of the linear oscillator model as a function of distance to the wall for a relaxation time, $\mathbf{b}$ delay, $\mathbf{c}$ gain and $\mathbf{d}$ expansion rate

of the visual surround (Paulus et al. 1989). For if this were the case, the expansion rate would always be at threshold and would not depend on distance as we found. Instead, this result indicates that the relation between visual motion and postural control must be viewed dynamically: the postural control system tends to minimize retinal slip, 
because the slip is smaller than when the subject does not move. However, this tendency also depends on the distance to the stimulus.

To examine the relationship of experimental results and model more quantitatively, we have estimated the order of magnitude of the model parameters. Such estimates can be based on the observed dependence of relaxation time, mean and angular deviation of relative phase on distance (cf. Eqs. 11, 15 and 17 in Schöner 1991). Proceeding in this manner, we found: $\alpha=0.5 \mathrm{~Hz}, \omega=2 \pi$ $0.18 \mathrm{rad} / \mathrm{s}, c_{e n v}=50 \mathrm{~cm} / \mathrm{s}$. In Fig. 10 we have plotted the resulting dependences of the various observables on distance. Clearly, amplitude is predicted to decrease much more strongly than observed, while all other relationships are captured quite well.

It is a general property of driven linear systems that the response amplitude decreases as the coupling to the driving force decreases. If we assume (as in Schöner 1991), that the visual expansion rate is the driving force, then relative timing stability decreases concomitantly. Therefore, the fact that this linkage of amplitude and stability is not observed in the present data hints that the theoretical picture of posture in a visual environment as a passive linear system driven by the expansion rate is not adequate. Instead, the central nervous system might actively generate movements (corresponding, mathematically, to non-linear dynamics possessing limit cycle attractors) which match the visual motion in amplitude and frequency. This active movement may then be coupled dynamically to the visual information, consistent with the successful model predictions for the timing aspects of the data. How compelling such an alternative theoretical view is will be tested in future experiments by manipulating the frequency of visual motion.

Acknowledgements. This work was supported by the Foundation for Biophysics, ESPRIT Basic Research Projects 3149 and 6615 and by MWF, Düsseldorf. We should like to thank Martin Giese, Erco Argante and Axel Steinhage for their assistance.

\section{References}

Asten WNJC van, Gielen CCAM, Denier van der Gon JJ (1988a) Postural adjustments induced by simulated motion of differently structured environments. Exp Brain Res 73:371-383

Asten WNJC van, Gielen CCAM, Denier van der Gon JJ (1988b) Postural movements induced by rotations of visual scenes. J Opt Soc Am [A] 5:1781-1789
Ball K, Pierowsky M (1987) A modified direct linear transformation method (DLT) calibration procedure to improve the accuracy of $3 \mathrm{~d}$ reconstruction for large volumes. In: Groot $\mathrm{G}$ de, Hollander AP, Huijing PA, Ingen Schenau GJ van (eds) Biomechanics XI$B$. (International series on biomechanics, vol 7-B), Free University, Amsterdam

Batschelet E (1981) Circular statistics in biology. Academic Press, London

Berthoz A, Lacour M, Soechting J, Vidal P (1979) The role of vision in the control of posture during linear motion. Prog Brain Res 50:197-209

Carter GC (1987) Coherence and time delay estimation. In: Lacoume JL, Durrani TS, Stora R (eds) Signal processing, vol 65. Les Houches, Session XLV. Elsevier, Amsterdam

Dichgans J, Brandt T (1978) Visual-vestibular interaction: effects on self-motion perception and postural control. In: Held R, Leibowitz HW, Teuber HL (eds) Perception (Handbook of sensory physiology, vol 8) Springer, Berlin Heidelberg New York

Dijkstra TMH, Gielen CCAM, Melis BJM (1992) Postural responses to stationary and moving scenes as a function of distance to the scene. Hum Movement Sci 11:195-203

Fisher SK, Ciufredda KJ (1988) Accommodation and apparent distance. Perception 17:609-621

Hays WL (1988) Statistics. Holt, Rinehart and Winston, Orlando, $\mathrm{Fa}$

Horn BKP (1987) Closed-form solution of absolute orientation using unit quaternions. J Opt Soc Am [A] 4:629-642

Lee DN (1980) The optic flow field: the foundation of vision. Philos Trans R Soc Lond [Biol] 290:169-179

Lee DN, Lishman JR (1975) Visual proprioceptive control of stance. J Hum Movement Stud 1:87-95

Lestienne F, Soechting J, Berthoz A (1977) Postural readjustments induced by linear motion of visual scenes. Exp Brain Res 28:363-384

Marmarelis PZ, Marmarelis VZ (1978) Analysis of physiological systems: the white-noise approach. Plenum Press, New York

Marple SL (1987) Digital spectral analysis with applications. Prentice Hall, Englewood Cliffs, NJ

Paulus W, Straube A, Krafczyk S, Brandt T (1989) Differential effects of retinal target displacement, changing size and changing disparity in the control of anterior/posterior and lateral body sway. Exp Brain Res 78:243-252

Schöner G (1991) Dynamic theory of action-perception patterns: the "moving room" paradigm. Biol Cybern 64:455-462

Schöner G, Kelso JAS (1988) Dynamic pattern generation in behavioural and neural systems. Science $239: 1513-1520$

Schuster D, Talbott RE (1980) Optimal and adaptive control in canine postural regulation. Am J Physiol 239: R93-R114

Stoffregen TA (1986) The role of optical velocity in the control of stance. Percept Psychophys 39:355-360

Tresilian JR (1991) Empirical and theoretical issues in the perception of time to contact. J Exp Psychol Hum Percept Perform $17: 865-876$ 